\title{
Fatores associados a ocorrência de depressão pós-parto: uma revisão sistemática
}

\author{
Factors associated with the occurrence of postpartum depression: a systematic review
}

Fasociados a la aparición de la depresión posparto: una revisión sistemática

\section{Resumo}

Objetivo: Analisar os principais fatores associados a ocorrência de depressão pós-parto. Metodologia: Revisão sistemática da literatura baseada no método Preferred Reporting Items for Systematic Reviews e Meta-Analysis (PRISMA), realizada nas bases de dados eletrônicas Pubmed, Scielo, Scopus e Web of Science, por meio dos seguintes descritores: "postpartum depression", "postnatal depression", "maternity blues", “puerperal depressive symptom", "after birth depression", "risk factors" e "dangerous factors". Foram incluídos artigos completos, disponíveis em português, inglês ou espanhol, publicados entre 2016 a 2021. Resultados: 12 artigos compuseram a amostra final deste estudo. Verificou-se que cinco eixos de fatores associados a depressão pós-parto foram identificados: sociodemográficos, psicológicos, sociais, hormonais e obstétricos. Grande parte destes fatores são interrelacionados e associados ao estilo de vida da mulher, especialmente os fatores sociais como baixo apoio social e familiar, conflito e insatisfação conjugal e exposição à violência por parceiro íntimo na gestação. Os fatores de risco mais frequentemente listados foram os físicos, incluindo desde a história de síndrome pré-menstrual a questões obstétricas como multiparidade, morbidade durante a gestação, gravidez não planejada, história de aborto espontâneo, parto cesáreo, pré-eclâmpsia, placenta prévia, descolamento prematuro de placenta, parto prematuro, hiperêmese gravídica, hipertensão gestacional e diabetes gestacional. Conclusão: Verificou-se que os fatores associados a 
depressão pós-parto podem ser sociodemográficos, psicológicos, sociais, hormonais e obstétricos. Portanto, os programas de prevenção desta condição clínica, como o pré-natal psicológico, precisam focar nas relações interpessoais dos indivíduos envolvidos, além dos fatores sociais e estilo de vida e das condições de saúde física destas mulheres.

Palavras-chave: Depressão pós-parto; Fatores de risco; Apoio social.

\begin{abstract}
Objective: To analyze the main factors associated with the occurrence of postpartum depression. Methodology: A systematic review of the literature based on the Preferred Reporting Items for Systematic Reviews and Meta-Analysis (PRISMA) method, carried out in the electronic databases Pubmed, Scielo, Scopus and Web of Science, using the following descriptors: "postpartum depression", "postnatal depression", "maternity blues", “puerperal depressive symptom", "after birth depression", "risk factors" and "dangerous factors". Full articles, available in Portuguese, English or Spanish, published between 2016 and 2021 were included. Results: 12 articles made up the final sample of this study. It was found that five axes of factors associated with postpartum depression were identified: sociodemographic, psychological, social, hormonal and obstetric. Most of these factors are interrelated and associated with the woman's lifestyle, especially social factors such as low social and family support, marital conflict and dissatisfaction, and exposure to intimate partner violence during pregnancy. The most frequently listed risk factors were physical, ranging from a history of premenstrual syndrome to obstetric issues such as multiparity, morbidity during pregnancy, unplanned pregnancy, history of miscarriage, cesarean delivery, preeclampsia, placenta previa, placental abruption, premature delivery, hyperemesis gravidarum, gestational hypertension and gestational diabetes. Conclusion: It was found that the factors associated with postpartum depression can be sociodemographic, psychological, social, hormonal and obstetric. Therefore, prevention programs for this clinical condition, such as psychological prenatal care, need to focus on the interpersonal relationships of the individuals involved, in addition to social factors and lifestyle and the physical health conditions of these women.
\end{abstract}

Keywords: Depression, postpartum; Risk factors; Social support.

\title{
Resumen
}

Objetivo: Analizar los principales factores asociados a la aparición de depresión posparto. Metodología: Revisión sistemática de la literatura basada en el método Preferred Reporting Items for Systematic Reviews and Meta-Analysis (PRISMA), realizada en las bases de datos electrónicas Pubmed, Scielo, Scopus y Web of Science, utilizando los siguientes descriptores: "postpartum depression", "postnatal depression", "maternity blues", "puerperal depressive symptom", "after birth depression", "risk factors" y "dangerous factors". Se incluyeron artículos completos, disponibles en portugués, inglés o español, publicados entre 2016 y 2021. Resultados: 12 artículos constituyeron la muestra final de este estudio. Se encontró que se identificaron cinco ejes de factores asociados a la depresión posparto: sociodemográficos, psicológicos, sociales, hormonales y obstétricos. La mayoría de estos factores están interrelacionados y asociados con el estilo de vida de la mujer, especialmente los factores sociales como el bajo apoyo social y familiar, el conflicto y la insatisfacción conyugales y la exposición a la violencia de pareja durante el embarazo. Los factores de riesgo enumerados con mayor frecuencia fueron físicos, incluidos desde antecedentes de síndrome premenstrual hasta problemas obstétricos como multiparidad, morbilidad durante el embarazo, embarazo no planificado, antecedentes de aborto espontáneo, parto por cesárea, preeclampsia, placenta previa, desprendimiento de placenta, parto prematuro, hiperémesis gravídica, hipertensión gestacional y diabetes gestacional. Conclusión: Se encontró que los factores asociados a la depresión posparto pueden ser sociodemográficos, psicológicos, sociales, hormonales y obstétricos. Por lo tanto, los programas de prevención de esta condición clínica, como el prenatal psicológico, necesitan enfocarse en las relaciones interpersonales de las personas involucradas, además de los factores sociales y de estilo de vida y las condiciones de salud física de estas mujeres.

Palabras clave: Depresión posparto; Factores de riesgo; Apoyo social.

\section{Introdução}

O período gravídico-puerperal é considerado de alto risco para o desenvolvimento de depressão e ansiedade devido às transformações em nível hormonal, físico e emocional vivenciadas pela mulher (WHO, 2009). Salienta-se que as mulheres são especialmente vulneráveis a transtornos psiquiátricos durante a gravidez e no período pós-parto e os transtornos de humor são os distúrbios psiquiátricos maternos mais comuns após o parto (Falana \& Carrington, 2019).

Os transtornos de humor pós-parto são frequentemente classificados em três categorias, em ordem crescente de gravidade: baby blues, depressão e psicose (O'Hara \& McCabe, 2013). A depressão pós-parto (DPP) é geralmente definida como um episódio de transtorno depressivo maior (embora a depressão menor às vezes seja incluída) que ocorre no período pós-parto (O'Hara \& McCabe, 2013). A prevalência de DPP varia de 1,9\% a 82,1\% em países desenvolvidos e de 5,2\% a 74\% 
em países em desenvolvimento e os sintomas depressivos persistem além do primeiro ano em $25 \%$ destas mulheres (Norhayati et al., 2015; Falana \& Carrington, 2019).

A DPP se manifesta como distúrbios do sono, alterações de humor, alterações no apetite, medo de lesões, sérias preocupações com o bebê, muita tristeza e choro, sensação de dúvida, dificuldade em concentração, falta de interesse nas atividades diárias, pensamentos de morte e suicídio; sentimentos de desesperança em casos graves do transtorno podem ameaçar a vida e levar ao suicídio (Aswathi et al., 2015; Norhayati et al., 2015).

Numerosos estudos mostraram que a DPP pode ter efeitos adversos tanto em mães quanto em crianças, de tal modo que as mães tinham duas vezes mais chances de sofrer de depressão durante quatro anos após o parto e também mais chances de apresentar doenças crônicas, além de apresentar uma qualidade de vida mais baixa no segundo e quarto meses pós-parto (Sadat et al., 2014; Abdollahi \& Zarghami, 2018). Ao mesmo tempo, alguns estudos apontaram os efeitos da DPP no desenvolvimento infantil, como: filhos de mães deprimidas tinham mais probabilidade de baixo peso e atrofia no primeiro ano de vida, além de apresentarem elevada incidência de problemas físicos (Gress-Smith et al., 2012; Farias-Antunez et al., 2018).

O modelo de vulnerabilidade-estresse explica como diferentes fatores podem afetar a suscetibilidade a distúrbios psicopatológicos; os fatores predisponentes são conceituados como fatores de risco ou fatores de proteção, dependendo se aumentam ou diminuem a probabilidade de um estressor levar à psicopatologia (Grant et al., 2008). A etiologia da DPP não é totalmente clara, de tal modo que até recentemente, pensava-se que este transtorno se devia principalmente a alterações biológicas após o parto; no entanto, o papel predominante dos fatores psicossociais no aumento do risco de desenvolver DPP é agora amplamente reconhecido (Gajaria \& Ravindran, 2018).

Neste sentido, é importante que o profissional de saúde esteja atento a sintomas que se configurem como mais desestruturantes e que fujam da adaptação "normal" característica do puerpério, que leve em conta a importância do acompanhamento no pós-parto imediato e no puerpério, prestando o apoio necessário à mulher no seu processo de reorganização psíquica quanto ao vínculo com o seu bebê, nas mudanças corporais e na retomada do planejamento familiar (Brasil, 2006).

Desta forma, determinar fatores que aumentam a probabilidade de desenvolver DPP ajudaria a identificar mulheres em risco, melhorando os esforços de prevenção e detecção precoce. Embora estudos anteriores tenham identificado vários fatores de risco para DPP, pesquisas recentes identificaram novos fatores. Desta forma, este estudo objetivou analisar os principais fatores associados a ocorrência de depressão pós-parto.

\section{Metodologia}

Trata-se de um estudo conduzido por meio de uma revisão sistemática da literatura, realizada com base no método Preferred Reporting Items for Systematic Reviews e Meta-Analysis (PRISMA) (Moher et al., 2015). A questão norteadora desta pesquisa incide em: "Quais os principais fatores associados a ocorrência de depressão pós-parto?"

Para a condução do estudo foi tomado como base as seguintes etapas idealizadas por Pati \& Lorusso (2018): 1) Definição da questão norteadora e objetivos da pesquisa; 2) Estabelecimento dos critérios de inclusão e exclusão/ amostragem dos estudos; 3) Busca na literatura; 4) Categorização e análise dos estudos; 5) Apresentação e discussão dos resultados da amostra e; 6) Apresentação e síntese do conhecimento.

A investigação dos estudos ocorreu nas bases de dados eletrônicas - Nacional Institute of Medicine (NIH-PubMed), Scopus, Scientific Electronic Library Online (Scielo) e Web of Science, no período de novembro de 2021 a janeiro de 2022. As bases de dados foram selecionadas pela sua grande abrangência de estudos. Para as buscas nestas bases, foram utilizados descritores extraídos do Medical Subject Headings (MeSH), a saber: "postpartum depression", "postnatal depression", "maternity blues", "puerperal depressive symptom", "after birth depression", "risk factors" e "dangerous factors". Foram 
utilizados os operadores booleanos “AND” e "OR” para o cruzamento dos descritores, com aplicações específicas para cada base de dados, conforme demonstrado no Quadro 1.

Quadro 1: Estratégia de busca nas bases de dados, Recife (PE), Brasil, 2022.

\begin{tabular}{|c|l|}
\hline $\begin{array}{c}\text { Base de dados } \\
\text { (artigos } \\
\text { recuperados) }\end{array}$ & \\
\hline PubMed (652) & $\begin{array}{c}((((((\text { postpartum depression) OR (postnatal depression)) OR (maternity blues)) OR (puerperal depressive symptom)) } \\
\text { OR (after birth depression)) AND (risk factors)) OR (dangerous factors) }\end{array}$ \\
\hline Scopus (332) & ( "puerperal depressive symptom" OR "postpartum depression" AND "risk factors" ) \\
\hline Scielo (68) & (postpartum depression AND risk factors) \\
\hline Web of Science & (postpartum depression OR after birth depression AND risk factors OR dangerous factors) \\
\hline
\end{tabular}

Fonte: Dados obtidos no estudo.

Os artigos selecionados atenderam aos seguintes critérios de inclusão: textos completos, disponíveis em português, inglês ou espanhol, publicados entre janeiro de 2016 a dezembro de 2021 e estudos com resultados baseados em dados estatísticos com associações entre as variáveis de interesse fornecidas. Foram excluídos artigos duplicados nas bases de dados (duplicatas), estudos que não enfocassem o puerpério, pesquisas com foco nos efeitos e tratamento da depressão pós-parto, estudos de revisão, editoriais, artigos de opinião, dissertações e teses e pesquisas com temática não relevante a questão norteadora da pesquisa.

Para garantir o registro conjunto de informações relevantes ao tema, foi utilizado o instrumento proposto por Nascimento et al. (2021), adaptado para este estudo com as seguintes variáveis: dados de identificação (título, autores, periódico, ano de publicação, país de origem do estudo, fator de impacto segundo o Journal Citation Reports - JCR, qualis e base de dados), delineamento metodológico (tipo/abordagem do estudo e nível de evidência) e principais resultados (número de participantes e fatores associados a ocorrência de DPP).

Após a aplicação dos filtros de pesquisa nas bases de dados, inicialmente foram encontrados 1.186 artigos. Os artigos duplicados (471) foram registrados apenas uma vez, totalizando em 715 para leitura dos títulos e resumos. Nesta etapa foram excluídas 514 publicações que não tinham abordagem relevante a temática deste estudo, 76 pesquisas voltadas apenas ao tratamento da DPP, 32 estudos de revisão e 27 artigos de opinião e dissertações. Sendo assim, 66 publicações foram selecionadas para leitura na íntegra, porém 42 foram excluídas por não apresentarem associações estatísticas nos resultados. Desta forma, 12 artigos constituíram a amostra final desta revisão, conforme explicitado na Figura 1. 
Figura 1: Fluxograma do processo de seleção do estudo, Recife (PE), Brasil, 2022.

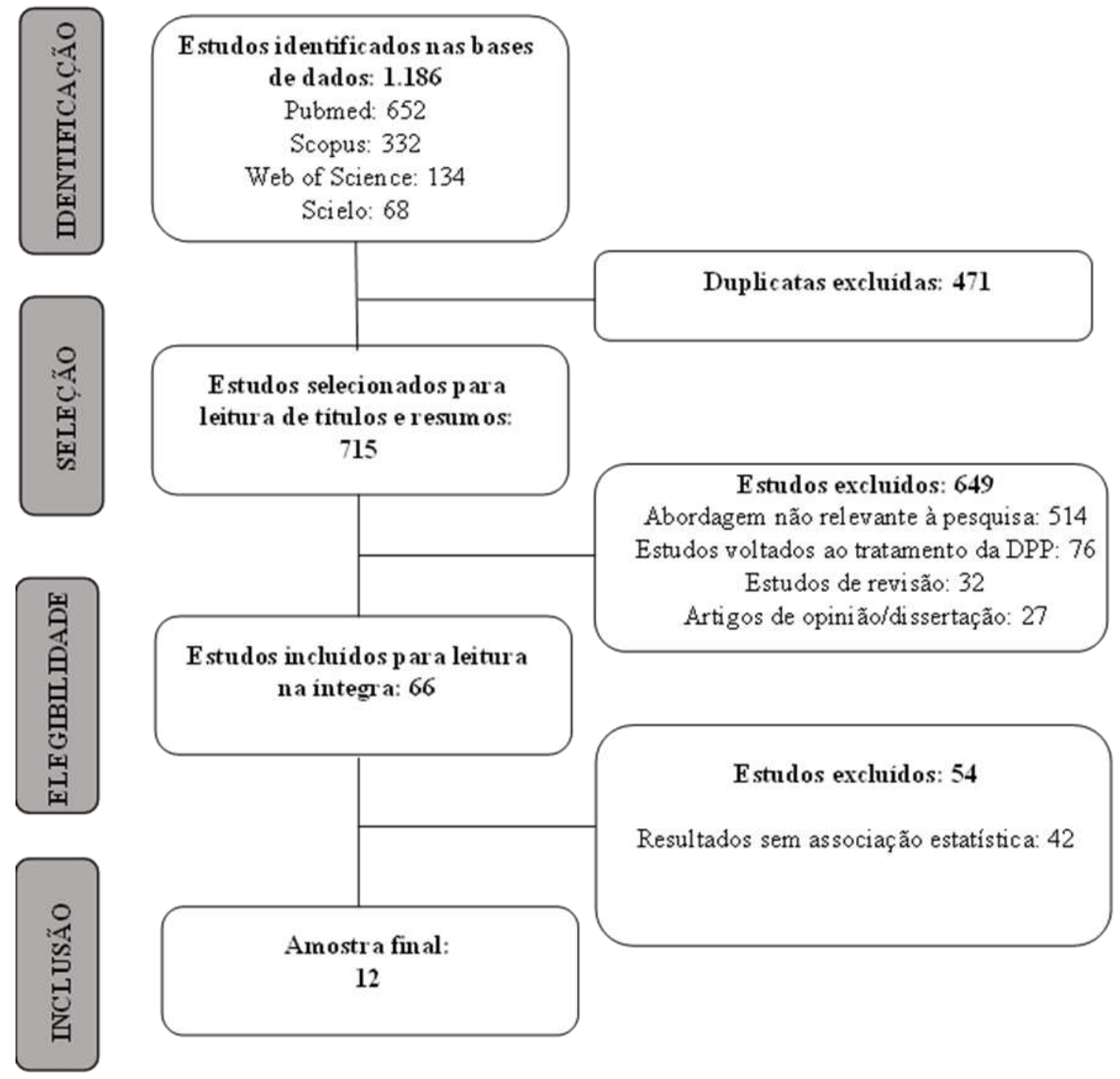

Fonte: Dados obtidos no estudo.

Os artigos selecionados foram submetidos à classificação do nível de evidência, a partir do instrumento de Classificação Hierárquica das Evidências para Avaliação dos Estudos (Stillwell et al., 2010). Segundo esta classificação, os níveis I e II são considerados evidências fortes, III e IV moderadas e V a VII fracas. Salienta-se que este estudo preservou os aspectos éticos de tal forma que todos os autores das publicações analisadas foram referenciados apropriadamente, mediante a Lei de Direitos Autorais no 9.610 de 19 de Fevereiro de 1998 (Brasil, 1998).

\section{Resultados}

Do total de 12 artigos analisados, observa-se, no Quadro 2, maior frequência de publicação em 2017 (n: 04) e maior produção do Brasil (n: 02) e Tailândia (n: 02). Em relação aos Qualis dos periódicos da amostra, sete estudos são classificados como A1 ou A2 pela CAPES. Quanto ao fator de impacto destes periódicos, o Psychological Medicine apresentou o JCR mais elevado (7,723). Referente à abordagem dos artigos encontrados, nove estudos são transversais, apresentando nível de evidência fraco (VI), enquanto quatro são estudos de coorte e um, caso controle, ambos apresentando nível de evidência moderado (III), mediante a classificação utilizada para análise. 
Quadro 2: Caracterização dos artigos da amostra final, Recife (PE), Brasil, 2022.

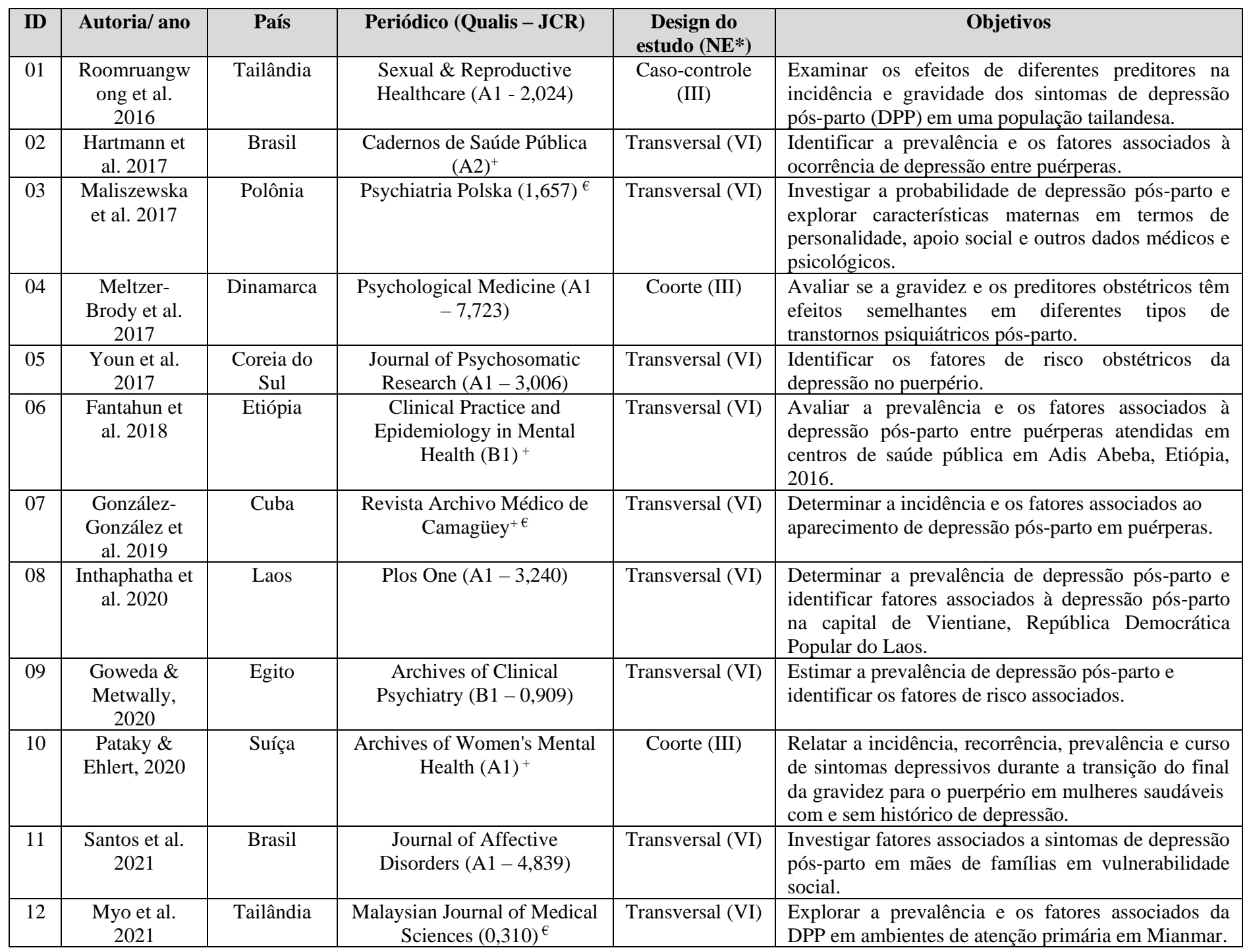

Nota: *NE - Nível de evidência; + Sem registro no JCR; € Sem registro de Qualis. Fonte: Dados obtidos no estudo.

Por meio do Quadro 3 é possível identificar os principais fatores associados a DPP nos estudos incluídos. No total, 1.670.493 puérperas foram incluídas nas análises. Verifica-se que cinco eixos de fatores foram identificados: sociodemográficos, psicológicos, sociais, físicos/hormonais e físicos/obstétricos.

Os principais fatores sociodemográficos associados estatisticamente a ocorrência de DPP foram: idade materna inferior a 20 anos e o estado civil solteiro. Referente aos fatores psicológicos, os principais elencados são: ter histórico de episódios depressivos pessoais anteriores à gestação, presença de estresse na gestação, presença de antecedentes psiquiátricos pessoais (neuroticismo), insatisfação com a gravidez e história familiar de transtornos mentais. Já os fatores de risco sociais foram: baixo apoio social e familiar, conflito e insatisfação conjugal e exposição à violência por parceiro íntimo na gestação.

A respeito dos fatores físicos, estes foram subdivididos em duas categorias - 1) Hormonal: história de síndrome prémenstrual; 2) Obstétrico: multiparidade, morbidade durante a gestação, gravidez não planejada, história de aborto espontâneo, parto cesáreo, pré-eclâmpsia, placenta prévia, descolamento prematuro de placenta, parto prematuro, hiperêmese gravídica, hipertensão gestacional e diabetes gestacional. 
Quadro 3: Fatores de risco associados a DPP, Recife (PE), Brasil, 2022.

\begin{tabular}{|c|c|c|}
\hline ID & $\begin{array}{c}\text { Número de } \\
\text { participantes }\end{array}$ & Fatores de risco \\
\hline 01 & 313 & $\begin{array}{l}\text { Multiparidade }(\mathrm{p}=0,009) \text {; histórico pessoal de depressão }(\mathrm{p}=\langle 0,0001) \text {; síndrome pré-menstrual }(\mathrm{p}= \\
0,042) \text {; baixo apoio social e familiar }(\mathrm{p}=0,012) \text {; recém-nascido não saudável }(\mathrm{p}=0,0005)\end{array}$ \\
\hline 02 & 2.687 & $\begin{array}{l}\text { Uso de álcool durante a gravidez }-{ }^{\epsilon} \mathrm{OR}=1,70(1,32-2,18) ; \mathrm{p}=0,001 ; \text { presença de morbidade na gestação } \\
-{ }^{\epsilon} \mathrm{OR}=4,27(3,43-5,31) ; \mathrm{p}=0,001\end{array}$ \\
\hline 03 & 387 & $\begin{array}{l}\text { Histórico de hospitalização na gravidez }-{ }^{\epsilon} \mathrm{OR}=3,51 ; * \mathrm{IC} 95 \%=1,32-9,20 ; \text { alto nível de neuroticismo - } \\
{ }^{\epsilon} \mathrm{OR}=1,37 ; * \text { IC } 95 \%=1,05-1,77\end{array}$ \\
\hline 04 & 392.458 & $\begin{array}{l}\text { Hiperêmese gravídica }-{ }^{\epsilon} \mathrm{OR}=2,69 ; * \mathrm{IC} 95 \%=1,93-3,73 ; \text { hipertensão gestacional }-{ }^{\epsilon} \mathrm{OR}=1,84 ; * \mathrm{IC} 95 \% \\
=1,33-2,55 ; \text { pré-eclâmpsia }-{ }^{\epsilon} \mathrm{OR}=1,45 ; * \mathrm{IC} 95 \%=1,14-1,84 ; \text { parto cesáreo }-{ }^{\epsilon} \mathrm{OR}=1,32 ; * \mathrm{IC} 95 \%= \\
1,13-1,53) \text {; estresse agudo na gestação e/ou pós-parto }-{ }^{\epsilon} \mathrm{OR}=1,93 ; * \mathrm{IC} 95 \%=1,38-2,71 ; \text { parto prematuro } \\
-{ }^{\epsilon} \mathrm{OR}=1,51 ; * \mathrm{IC} 95 \%=1,30-1,75 ; \text { diabetes gestacional }-{ }^{\epsilon} \mathrm{OR}=1,42 ; * \mathrm{IC} 95 \%=1,03-1,97\end{array}$ \\
\hline 05 & 1.269 .130 & $\begin{array}{l}\text { História anterior de depressão }-{ }^{\epsilon} \mathrm{OR}=16,72 ; * \mathrm{IC} 95 \%=16,05-17,41 ; \text { idade materna mais jovem }(<20 \\
\text { anos })-{ }^{\epsilon} \mathrm{OR}=1,6 ; * \mathrm{IC} 95 \%=1,39-1,85 ; \text { parto cesáreo }-{ }^{\epsilon} \mathrm{OR}=1,33 ; * \mathrm{IC} 95 \%=1,28-1,38 ; \text { pré-eclâmpsia } \\
-{ }^{\epsilon} \mathrm{OR}=1,12 ; * \mathrm{IC} 95 \%=1,03-1,22 ; \text { diabetes gestacional }-{ }^{\epsilon} \mathrm{OR} 1,04 ; * \text { IC95\% }=0,98-1,11 ; \text { placenta prévia } \\
-{ }^{\epsilon} \mathrm{OR}=0,96 ; * \mathrm{IC} 95 \%=0,83-1,11 ; \text { descolamento prematuro de placenta }-{ }^{\epsilon} \mathrm{OR}=1,33 ; * \mathrm{IC} 95 \%=1,10- \\
1,62 ; \text { parto prematuro }-{ }^{\epsilon} \mathrm{OR}=1,41 ; * \mathrm{IC} 95 \%=1,31-1,52\end{array}$ \\
\hline 06 & 633 & $\begin{array}{l}\text { Ser solteira }-{ }^{\epsilon} \mathrm{OR}=7,7 ; * \mathrm{IC} 95 \%=3,0-19,5 ; \text { história pregressa de óbito infantil }-{ }^{\epsilon} \mathrm{OR}=2,7 ; * \mathrm{IC} 95 \%= \\
1,6-4,4 ; \text { desemprego }-{ }^{\epsilon} \mathrm{OR}=2,9 ; * \mathrm{IC} 95 \%=1,3-6,4 ; \text { parto sem a presença de familiares }-{ }^{\epsilon} \mathrm{OR}=5,5 ; \\
* \mathrm{IC} 95 \%=2,6-11,6 ; \text { história de uso de substâncias }-{ }^{\epsilon} \mathrm{OR}=2,6 ; * \mathrm{IC} 95 \%=1,4-4,8\end{array}$ \\
\hline 07 & 139 & $\begin{array}{l}\text { Idade inferior a } 20 \text { anos }\left({ }^{\epsilon} \mathrm{OR}=1,55\right) \text {; estado civil solteiro }\left({ }^{\epsilon} \mathrm{OR}=11,16\right) \text {; multiparidade }\left({ }^{\oplus} \mathrm{OR}=5,66\right) \text {; } \\
\text { história de depressão anterior à gravidez }\left({ }^{€} \mathrm{OR}=5,45\right) \text {; história de violência por parceiro íntimo na } \\
\text { gestação }\left({ }^{€} \mathrm{OR}=4,71\right)\end{array}$ \\
\hline 08 & 428 & $\begin{array}{l}\text { Gravidez indesejada }-{ }^{\epsilon} \mathrm{OR}=1,66 ; * \mathrm{IC} 95 \%=1,00-2,73 ; \mathrm{p}=0,049 ; \text { histórico de depressão durante a } \\
\text { gravidez }-{ }^{\epsilon} \mathrm{OR}=3,99 ;{ }^{*} \mathrm{IC} 95 \%=2,35-6,77 ; \mathrm{p}<0,001 ; \text { insatisfação conjugal }-{ }^{\epsilon} \mathrm{OR}=1,88 ; * \mathrm{IC} 95 \%= \\
0,99-3,56 ; \mathrm{p}=0,053\end{array}$ \\
\hline 09 & 237 & $\begin{array}{l}\text { Gravidez indesejada }(p=0,000) ; \text { multiparidade }(p=0,004) \text {; recém-nascido não saudável }(p=0,018) \text {; } \\
\text { conflito conjugal }(p=0,000)\end{array}$ \\
\hline 10 & 687 & $\begin{array}{l}\text { Histórico de depressão - }{ }^{\epsilon} \mathrm{OR}=2,254 ; * \mathrm{IC} 95 \%=1,259-4,035 ; \text { sintomas de síndrome pré-menstrual antes } \\
\text { da gravidez }-{ }^{\epsilon} \mathrm{OR}=2,828 ; * \mathrm{IC} 95 \%=1,337-5,983\end{array}$ \\
\hline 11 & 3.174 & Multiparidade $(\mathrm{p}<0,001)$ \\
\hline 12 & 220 & Gravidez não planejada $-{ }^{\epsilon} \mathrm{OR}=2,946 ;$ menos de quatro consultas de pré-natal $-{ }^{\epsilon} \mathrm{OR}=2,518$ ) \\
\hline
\end{tabular}

Notas: *IC95\% - Intervalo de confiança de 95\%; €OR - Odds Ratio (Razão de probabilidade). Fonte: Dados obtidos no estudo.

\section{Discussão}

Este estudo analisou a produção científica a respeito dos principais fatores associados a ocorrência de DPP. Por meio dos estudos incluídos na análise, observou-se que estes fatores podem ser subdivididos em cinco categorias interrelacionadas: sociodemográficos, psicológicos, sociais, físicos/hormonais e físicos/obstétricos.

A respeito dos fatores sociodemográficos, houve vários achados sobre a associação da idade materna (inferior a 20 anos) e DPP nos estudos. Neste limiar, as mães adolescentes são conhecidas como uma das populações vulneráveis para DPP (Liu et al. 2018). As mães adolescentes têm dificuldades não apenas com seu papel materno, mas também com várias mudanças psicológicas e físicas em si mesmas (Silverman et al., 2017). Estudos anteriores também relataram que a idade materna avançada pode estar associada a complicações maternas e fetais na gravidez, como trabalho de parto disfuncional, aborto espontâneo, natimorto e parto de recém-nascidos pequenos ou grandes para a idade gestacional; estes podem contribuir para o desenvolvimento de depressão durante o período pós-parto (Dakov \& Dimitrova, 2014; Vanya et al., 2015).

Em relação aos fatores de risco psicológicos associados a DPP, vários estudos da amostra apresentaram dados estatísticos a respeito destes elementos. Constata-se que história prévia de depressão e ansiedade está entre os fatores que estão associados a um maior risco de DPP. A ocorrência de distúrbios de saúde mental, como depressão durante a gravidez é um fator poderoso na previsão de DPP (Lancaster et al., 2010). Há evidências para explicar essas relações sugerindo que mulheres com histórico positivo de depressão são mais suscetíveis a alterações hormonais, afetando o início da DPP (Buttner et al., 2013). Em mulheres com síndrome pré-menstrual grave, por exemplo, o sistema de transporte de serotonina mudará enquanto a área de polimorfismo do transportador de serotonina está associada à depressão maior; p polimorfismo de serotonina elevado 
pode levar à depleção de triptofano e indução de maior DPP (Sanjuan et al., 2008).

Fatores sociais/familiares também foram identificados como associados a ocorrência de DPP. Nesta perspectiva, salienta-se que apoio social se refere a apoio emocional, apoio financeiro, apoio de inteligência e relações de empatia. O papel do apoio social na redução da DPP já foi demonstrado cientificamente (Escribà-Agüir \& Artazcoz, 2011). Reduzir o apoio social é o fator ambiental mais característico como preditor para o início da depressão e transtornos de ansiedade (LandmanPeeters et al., 2005).

Fatores de risco hormonais também foram identificados em alguns estudos da amostra (Roomruangwong et al., 2016; Pataky \& Ehlert, 2020). Estas pesquisas indicaram uma associação entre a síndrome pré-menstrual e a ocorrência de DPP. Esta síndrome se trata de um distúrbio que corresponde a sintomas físicos, comportamentais e emocionais que ocorrem durante a fase lútea tardia do ciclo menstrual, podendo impactar significativamente a qualidade de vida das mulheres ao longo de seus anos reprodutivos (Stute et al., 2017). Tem-se a hipótese de que os sintomas apresentados durante esta fase são consequência de uma vulnerabilidade afetiva às flutuações hormonais, que ocorrem não apenas no período pré-menstrual, mas também no pós-parto. Essa vulnerabilidade pode ser entendida como uma resposta biológica a alterações hormonais que podem levar à depressão, devido ao efeito de mudanças repentinas nos níveis de progesterona e estrogênio em regiões do cérebro associadas à regulação do humor (Corwin et al., 2015).

Referente aos fatores de risco obstétricos, estes estiveram presentes em grande parte dos estudos incluídos nos resultados desta revisão. Neste contexto, a avaliação da relação entre o número de partos e a DPP tem sido associada a resultados conflitantes. Um estudo realizado com 86 participantes dentro de 6 semanas após o parto descobriu que ter dois ou mais filhos devido à maior carga psicológica é mais provável de estar associado à ocorrência de depressão (Mathisen et al., 2013). As discrepâncias entre os resultados desses estudos sugerem que o número de partos por si só não é um fator independente para desenvolver a DPP e o desenvolvimento de condições patológicas para a ocorrência do adoecimento é causado por condições psicossociais que a multiplicidade de partos cria para a mulheres (Santos et al., 2021).

A pré-eclâmpsia foi um dos preditores de DPP analisados nesta revisão. Pesquisadores relataram que alterações físicas e hormonais podem estar relacionadas a essas associações. Sabe-se que os níveis de serotonina no sangue aumentam em mulheres grávidas com pré-eclâmpsia, enquanto os níveis de serotonina no cérebro diminuem, o que pode levar a sintomas depressivos (Koutra et al., 2016). O parto prematuro também foi identificado como um preditor da DPP; situações como esta podem causar problemas como complicações médicas, temperamento difícil, distração e excitação negativa em recém-nascidos prematuros. Além disso, a própria experiência do parto prematuro pode estar relacionada ao trauma psicológico para as mães, de tal modo que esses fatores podem aumentar a carga materna e o estresse psicológico (Chang et al., 2016).

O descolamento de placenta também proporciona experiências traumáticas para as mães. Pesquisadores reconhecem que os sintomas e complicações do descolamento prematuro da placenta influenciam a saúde mental materna, incluindo a depressão durante o período pós-parto (Cunningham et al., 2014). Dor abdominal de início súbito, aumento e contrações uterinas, sangramento vaginal e diminuição do movimento fetal são sintomas comuns desta condição clínica. Em alguns casos, ela pode levar a condições com risco de vida, incluindo anormalidades da frequência cardíaca fetal, hemorragia materna grave e coagulação intravascular disseminada. Estas são experiências traumáticas que podem levar as mulheres a ter depressão (Cunningham et al., 2014).

Desta forma, diante do conhecimento dos fatores associados a ocorrência de DPP, instituir formas de prevenção por meio dos profissionais de saúde, especialmente da Atenção Primária à Saúde, é de extrema importância. Neste contexto, o prénatal psicológico, que vem inovando com o conceito em atendimento perinatal aprimorado, tem se tornado um grande aliado neste processo. O programa tem como o objetivo a integração da gestante e da família a todo o processo da gravidez e pós gravidez, oferecendo apoio emocional e discutindo situações de dúvidas que podem surgir durante este ciclo, como mitos 
relacionados a maternidade, gestação de risco, à malformação do feto, medo do parto, transtornos depressivos e de ansiedade, às mudanças de papéis familiares e sociais, às alterações na libido, entre outros (Souza et al., 2021).

\section{Conclusão}

Verificou-se que os fatores associados a DPP podem ser classificados em cinco categorias, desde fatores sociodemográficos a fatores psicológicos, sociais, hormonais e obstétricos. De acordo com os achados deste estudo, muitos fatores biológicos e ambientais, como fatores relacionados ao estilo de vida, estão envolvidos na incidência ou prevenção da DPP por meio de impacto direto e indireto sobre o nível de serotonina no cérebro e sua função. Além disso, muitos fatores ambientais, como fatores socioeconômicos, causam condições de crise e DPP, influenciando a saúde mental durante a gravidez.

Portanto, os programas de prevenção da DPP, como o pré-natal psicológico, precisam focar nas relações interpessoais dos indivíduos para reduzir a violência doméstica e aumentar a proteção social, além de modificar a vida da mulher, estilo de vida e aumentar sua capacidade de lidar com as condições de crise. Além disso, com base nos resultados desta pesquisa, as ferramentas preditoras de DPP devem focar fatores sociais e estilo de vida, além das condições de saúde física dos indivíduos envolvidos.

\section{Referências}

Abdollahi, F. \& Zarghami, M. (2018). Effect of postpartum depression on women's mental and physical health four years after childbirth. East. Mediterr. Health J. 24(10), 1002-1009.

Aswathi, A., Rajendiren, S., Nimesh, A., Philip, R. R., Kattimani, S., Jayalakshmi, D., et al. (2015). High serum testosterone levels during postpartum period are associated with postpartum depression. Asian J Psychiatr. 17, 85-8.

Buttner, M. M., Mott, S. L., Pearlstein, T., Stuart, S., Zlotnick, C. \& O'Hara, M. W. (2013). Examination of premenstrual symptoms as a risk factor for depression in postpartum women. Arch Womens Ment Health. 16(3), 219-225.

Brasil. Ministério da Saúde (MS). (1998). Lei no 9.610, de 19 de fevereiro de 1998, que altera, atualiza e consolida a legislação sobre direitos autorais e dá outras providências. Diário Oficial da União. Brasília: Ministério da Saúde. Recuperado de http://www.planalto.gov.br/ccivil_03/leis/19610.htm

Brasil. Ministério da Saúde (MS). (2006). Secretaria de Atenção à Saúde. Departamento de Ações Programáticas Estratégicas. Área Técnica de Saúde da Mulher. Pré-natal e Puerpério: atenção qualificada e humanizada - manual técnico/Ministério da Saúde. Secretaria de Atenção à Saúde, Departamento de Ações Programáticas Estratégicas - Brasília: Ministério da Saúde, 163.

Chang, H.-P., Chen, J.-Y., Huang, Y.-H., Yeh, C.-J., Huang, J.-Y., Su P-H, et al. (2016). Factors associated with post-traumatic symptoms in mothers of preterm infants. Arch. Psychiatr. Nurs. 30(1), 96-101.

Corwin, E. J., Pajer, K., Paul, S., Lowe, N., Weber, M. \& McCarthy, D.O. Bidirectional psychoneuroimmune interactions in the early postpartum period influence risk of postpartum depression. Brain, Behavior, and Immunity. 49, 86-93.

Cunningham, F. G., Leveno, K. J., Bloom, S. L., Spong, C. Y., Dashe, J. S., Hoffman, B. L., et al. (2014). Williams Obstetrics. (24a ed.), McGraw-Hill Education, Columbus.

Dakov, T. \& Dimitrova, V. (2014). Pregnancy and delivery in women above the age of 35. Akush Ginekol (Sofiia). 53(1), 13-20.

Falana, S. D. \& Carrington, J. M. (2019). Postpartum Depression: are You Listening? Nurs. Clin. North Am. 54(4), $561-567$.

Fantahun. A., Cherie, A. \& Deribe, L. (2018). Prevalence and Factors Associated with Postpartum Depression Among Mothers Attending Public Health Centers of Addis Ababa, Ethiopia, 2016. Clin Pract Epidemiol Ment Health. 14, 196-206.

Farias-Antunez, S., Xavier, M. O. \& Santos, I. S. (2018). Effect of maternal postpartum depression on offspring's growth. J. Affect. Disord. 228 , 143-152.

Gajaria, A. \& Ravindran, A. V. (2018). Interventions for perinatal depression in low and middle-income countries: a systematic review. Asian J. Psychiatr. 37, $112-120$.

González-González, A., Casado-Mendez, P., Molero-Segrera, M. \& López-Sánchez. et al. (2019). Associated factors for postpartum depression. Revista Archivo Médico de Camagüey; 23(6), 770-779.

Goweda, R. \& Metwally, T. (2020). Prevalence and associated risk factors of postpartum depression: a cross sectional study. Arc. Clin. Psychiatr. 47(4), 106109.

Grant, K. A., McMahon, C. \& Austin, M. P. (2008). Maternal anxiety during the transition to parenthood: a prospective study. J. Affect. Disord. 108, 101-111. 
Gress-Smith, J. L., Luecken, L. J., Lemery-Chalfant, K. \& Howe, R. (2012). Postpartum depression prevalence and impact on infant health, weight, and sleep in low-income and ethnic minority women and infants. Matern. Child Health J. 16, 887-893.

Inthaphatha, S., Yamamoto, E., Louangpradith, V., Takahashi, Y., Phengsavanh, J., Kariya, T., et al. (2020). Factors associated with postpartum depression among women in Vientiane Capital, Lao People's Democratic Republic: A cross-sectional study. Plos One; 15(12), e0243463.

Koutra, K., Vassilaki, M., Georgiou, V., Koutis, A., Bitsios, P., Kogevinas, M., et al. (2016). Pregnancy, perinatal and postpartum complications as determinants of postpartum depression: the Rhea mother-child cohort in Crete, Greece. Epidemiol. Psychiatr. Sci. $27(3), 1-12$.

Lancaster, C. A., Gold, K. J., Flynn, H. A., Yoo, H., Marcus, S. M. \& Davis, M. M. (2010). Risk factors for depressive symptoms during pregnancy: A systematic review. Am J Obstet Gynecol. 202(1), 5-14.

Liu, S., Yan, Y., Gao, X., Xiang, S., Sha, T., Zeng, G., et al. (2017). Risk factors for postpartum depression among Chinese women: path model analysis. BMC Pregnancy Childbirth. 17, 133.

Maliszewska, K., Bidzan, M., Świątkowska-Freund, M. \& Krzysztof, P. (2017). Screening for maternal postpartum depression, and associations with personality traits and social support. A Polish follow-up study 4 weeks and 3 months after delivery. Psychiatr. Pol., 51(5), 889-898.

Mathisen, S. E., Glavin, K., Lien, L. \& Lagerløv, P. (2013). Prevalence and risk factors for postpartum depressive symptoms in Argentina: a cross-sectional study. Int J Womens Health. 5, 787-93.

Meltzer-Brody, S., Maegbaek, M. L., Medland, S. E., Miller, W. C., Sullivan, P. \& Munk-Olsen, T. (2017). Obstetrical, pregnancy and socio-economic predictors for new-onset severe postpartum psychiatric disorders in primiparous women. Psychol Med. 47(8), 1427-1441.

Moher, D., Shamseer, L., Clarke, M.; Ghersi, D., Liberati, A., Petticrew, M., et al (2015). Preferred reporting items for systematic review and meta-analysis protocols (PRISMA-P) 2015 statement. Syst. Rev. 4(1), 1-9.

Myo, T., Hong, S. A., Thepthien, B. O. \& Hongkrailert, N. (2021). Prevalence and Factors Associated with Postpartum Depression in Primary Healthcare Centres in Yangon, Myanmar. Malays J Med Sci; 28(4), 71-86.

Nascimento, J. W. A., Santos, R. S., Santos, T. M. R., Silva, A. L. B., Rodrigues, L. D. C., Silva, V. W., et al. (2021). Complications associated with intimate partner violence in pregnant women: a systematic review. Int. J. Dev. Res. 11(7), 48924-48928.

Norhayati, M. N., Hazlina, N. H., Asrenee, A. R. \& Emilin, W. M. (2015). Magnitude and risk factors for postpartum symptoms: a literature review. J. Affect. Disord. 175, 34-52.

O’Hara, M. W. \& Mc Cabe, J. E. (2013). Postpartum depression: current status and future directions. Annu. Rev. Clin. Psychol. 9, $379-407$.

Pataky. E. A. \& Ehlert, U. (2020). Longitudinal assessment of symptoms of postpartum mood disorder in women with and without a history of depression. Arch Womens Ment Health; 23(3), 391-399.

Roomruangwong, C., Withayavanitchai, S. \& Maes, M. (2016). Antenatal and postnatal risk factors of postpartum depression symptoms in Thai women: A case-control study. Sex Reprod Healthc. 10, 25-31.

Sadat, Z., Abedzadeh-Kalahroudi, M., Kafaei Atrian, M., Karimian, Z. \& Sooki, Z. (2014). The impact of postpartum depression on quality of life in women after child's birth. Iran. Red Crescent Med. J. 16(2), e14995.

Sanjuan, J., Martin-Santos, R., Garcia-Esteve, L., Carot, J. M., Guillamat, R., Gutierrez-Zotes, A., et al. (2008). Mood changes after delivery: role of the serotonin transporter gene. Br J Psychiatry. 193(5), 383-388.

Santos, I. S., Munhoz, T. N., Blumenberg, C., Barcelos, R., Bortolotto, C. C., Matijasevich, A., et al. (2021). Post-partum depression: a cross-sectional study of women enrolled in a conditional cash transfer program in 30 Brazilian cities. J Affect Disord; 281, 510-516.

Silverman, M. E., Reichenberg, A., Savitz, D. A., Cnattingius, S., Lichtenstein, P., Hultman, C. M., et al. (2017). The risk factors for postpartum depression: a population-based study. Depress Anxiety. 34(2), 178-187.

Souza, N. K. P., Magalhães, E. Q. \& Rodrigues Junior, O. M. (2021). A prevalência da depressão pós-parto e suas consequências em mulheres no Brasil. Research, Society and Development. 10(15), e597101523272.

Stillwell, S., Fineout-Overholt, E., Melnyk, B. M. \& Wiliamson, K. M. (2010). Evidence- based practice: step by step. Am J Nurs; $110(5)$, $41-47$.

Stute, P., Bodmer, C., Ehlert, U., Eltbogen, R., Ging, A., Streuli, I., et al. (2017). Interdisciplinary consensus on management of premenstrual disorders in Switzerland. Gynecological Endocrinology. 33(5), 342-348.

Vanya, M., Szili, K., Devosa, I. \& Bartfai, G. (2015). Risk of maternal and fetal disease among women older than 40 years. Orv. Hetil. 156(49), 1987-1990.

World Health Organization (WHO). (2009). United Nations Population Fund. Mental health aspects of women's reproductive health. A global review of the literature. Geneva: WHO Press.

Youn, H., Lee, S., Han, S. W., Kim, L. Y., Lee, T. S., Oh, M. J., et al. (2017). Obstetric risk factors for depression during the postpartum period in South Korea: a nationwide study. J Psychosom Res. 102, 15-20. 